\title{
The role of reactive flow in driving melt fraction change and chemical differentiation in mush reservoirs
}

\author{
HAIYANG HU ${ }^{1}$, MATTHEW D. JACKSON ${ }^{1}$ AND \\ JONATHAN D. BLUNDY ${ }^{2}$ \\ ${ }^{1}$ Imperial College London \\ ${ }^{2}$ Oxford University \\ Presenting Author: h.hu@imperial.ac.uk
}

To understand and explain the formation of chemically evolved, low crystallinity magmas, it is necessary to understand the processes that cause, and control, changes in melt fraction and composition in crustal mush reservoirs. Melt fraction change by heating or cooling is well understood and widely invoked but does not yield differentiation, because there is no separation of melt and solid crystals. Fractional crystallisation at high melt fraction is typically invoked to explain differentiation, but is inconsistent with the evidence that large-scale, long-term magma storage and evolution occurs at low melt fraction.

At low melt fraction, relative flow of melt and matrix to yield melt fraction change and differentiation is widely assumed to occur by compaction. Yet textural evidence for crystal deformation consistent with compaction at low melt fraction is scarce. Consequently, it is not straightforward to explain how melt fraction and composition change in crustal mush reservoirs: heating and cooling alone do not yield composition change, while compaction yields both, but is not obviously consistent with the rock record.

Here we show that melt fraction change can be expressed in terms of heating/cooling and compaction, plus an additional term that does not correspond to either of these processes and which we term 'reactive flow'. Similarly, we show that composition change can be expressed in terms of compaction and reactive flow; heating/cooling does not contribute. We quantify the relative contributions of these different mechanisms to melt fraction and composition change in a crustal mush reservoir using numerical modelling and a number of example cases.

We show that reactive flow acts to oppose melt fraction changes caused by compaction, maintaining melt channels for ongoing loss of melt. Reactive flow also causes component exchange between solid and melt, which drives differentiation in compacting parts of the reservoir to produce more refractory bulk composition. The contribution of reactive flow in driving differentiation is similar in magnitude to that of compaction and can explain the relative lack of evidence for compaction in the igneous rock record: refractory compositions are produced by reactive flow at moderate melt fraction, rather than compaction to very low melt fraction. 